Manuelle Medizin 2011 · 49:471-472 DOI 10.1007/s00337-011-0893-0

Online publiziert: 26. November 2011

(c) Springer-Verlag 2011

\author{
H. Locher ${ }^{1}$ • U. Böhni' ${ }^{2}$ J. Buchmann ${ }^{3}$ - M. Habring ${ }^{4}$. W. von Heymann ${ }^{5}$ \\ ${ }^{1}$ Zentrum für Schmerztherapie und manuelle Medizin, Tettnang \\ 2 ZeniT Zentrum für interdisziplinäre Therapie des Bewegungsapparates, Schaffhausen \\ ${ }^{3}$ Klinik für Psychiatrie, Neurologie, Psychosomatik und Psychotherapie \\ im Kindes- und Jugendalter, Universität Rostock \\ ${ }^{4}$ Praxis für Allgemeinmedizin, Bad Ischl \\ ${ }^{5}$ Orthopädische Praxis, Bremen
}

\title{
Manuelle Medizin und translationale Forschung
}

Reihe von anatomischen Studien, die kontralaterale Projektionen von nozizeptiven Afferenzen auf trigeminale und zervikale Hinterhornneurone beschreiben, rücken mehr und mehr in den Blickpunkt des Interesses. ... Diese anatomische Anordnung von Neuronen möchte ihr Korrelat in den schwer zu lokalisierenden dumpfen Kopfund Nackenschmerzen finden" [2].

Die translationale Vorgehensart findet sich auch umfangreich in zurückliegenden Jahrhunderten, insbesondere bei Descartes und seinem Modell der Schmerzperzeption auf gänzlich mechanische Modellgedanken [3] und auch sehr umfangreich in den osteopathischen Erklärungsansätzen für verschiedenste Phänomene auf dem Boden makroanatomischer Details [1].

Eine Erklärung dafür, dass das tatsächliche Verhalten des Modells „Homo sapiens“ z. T. auch durchaus von den Erwartungen, die die Resultate aus dem Bereich Katze und kleine Nager betreffen, abweicht, mag in der wichtigen und entwicklungsgeschichtlich spät eingetretenen Rolle des humanen Großhirns liegen. Unbenommen ist aber die im Vergleich wesentlich größere Summe der anatomischen und neurophysiologischen Übereinstimmungen, die auch in allen anderen Fächern fraglos große Mengen an völlig zutreffenden Analogieschlüssen gestatten. Dies ist ein wichtiger Grund dafür, eine so umfangreiche Grundlagenforschung, wie sie derzeit weltweit geschieht, auch zu rechtfertigen [1].

Die Untermauerung der Praxis translationaler Rückschlüsse und entsprechend evidenzbasierter, mit klinischen Beobachtungen kongruenter Inhalte soll mittelfristig folgende Effekte zeitigen können:

- Befreiung von exklusiv empirischen, mehr oder weniger fragwürdigen, auch historisch bedingten Erklärungsmodellen,

- Präzisierung und Herstellung einer Begründbarkeit für diagnostische und therapeutische Ansätze, insbesondere langfristige therapeutische Strategien im Bereich Schmerztherapie unter Einbezug der Techniken der manuellen Medizin,

- Manifestation naturwissenschaftlicher Erklärungsmodelle für klinisch empirische Phänomene bei gleichzeitiger Abkehr von paramedizinischer Spekulation oder überwiegend philosophischen Projektionen auf das ärztliche Behandeln.

Das ärztliche Handeln ist natürlich auch im Kontext philosophischer Projektionen täglich zu hinterleuchten. In Modellen zur Therapieplanung soll aber in der heutigen Zeit die sog. harte Wissenschaft die deutliche und nachvollziehbare Oberhand behalten. Schließlich sollten Wirkungsweise, Indikationsstellung und technisch-therapeutische Anwendungen der manualmedizinischen und/oder osteopathischen Verfahrenstechniken eine rationale Verankerung in der wissenschaftlichen Medizin erfahren. Nur so können sie Bestandteil einer wissenschaftlich orientierten und „evidenzierbar“ effektiven schmerztherapeutischen Versorgung auf dem Bozur Erklärung klinischer Zustandsbilder aus anatomischen Gegebenheiten: „Eine 


\section{Weiterführende Literatur}

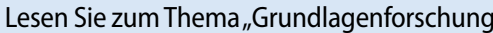
trifft Manualmedizin" auch die Beiträge:

- Heymann W von, Böhni U, Locher H (2005) Grundlagenforschung trifft Manualmedizin. Ergebnisse der Bodenseekonferenz deutschsprachiger Manualmediziner, 22.-24. Juli 2005, Bad Horn, Schweiz. Manuelle Med 43:385-394

- Neuhuber W (2005) M. longissimus als Vermittler zwischen kraniozervikalem Übergang und Becken. Manuelle Med 43:395-399

den einer substanziellen Schmerzanalyse [5] werden.

Bei den von der Arbeitsgruppe Böhni, Buchmann, Habring, von Heymann und Locher initiierten Bodenseetagungen in Bad Horn (Thurgau, Schweiz) unter dem Titel „Grundlagenforschung trifft Manualmedizin“ wurde im ausführlichen Dialog mit bekannten Grundlagenforschern (Prof. Dr. S. Mense, Institut für Zellbiologie, Universität Heidelberg; Prof. Dr. J. Sandkühler, Institut für Hirnforschung, Universität Wien; Prof. Dr. W. Neuhuber, Anatomisches Institut der Universität Erlangen; Prof. Dr. W. Zieglgänsberger, Max-Planck-Institut für Psychiatrie, München; Prof. Dr. W. Magerl, Physiologisches Institut, Universität Mannheim) sowie anderen namhaften Psychiatern, Neurologen und Psychologen umfangreiches Material gesammelt. Dieses konnte durch die Arbeitsgruppe auf die klinisch empirischen Phänomene der täglichen Arbeit übertragen und mit grundlagenwissenschaftlicher Entsprechung belegt werden.

Die daraus abgeleiteten klinischen Hypothesen, die in den Texten der kommenden Artikelserie farblich und gestalterisch hervorgehoben sein werden, sollen als Interpretationen im Sinne der Projektion von Ergebnissen der Grundlagenforschung sowie der Ergebnisse psychologischer und psychiatrischer Forschung auf diejenigen physischen Phänomene verstanden werden, die in der täglichen Praxis im Umgang mit manualmedizinischen und osteopathischen Techniken beobachtet werden. Aus der Umsetzung dieser auf die Grundlagenforschung bezogenen Hypothesen, ihrer Pathophysiologie, ihrer Membranbiochemie und ihrer makro- und mikroanatomischen Korrelate sollen bei der Diagnostik komplexer funktioneller Störungsbilder und bei der Indikationsstellung gezielter therapeutischer Maßnahmen Hilfestellung geben.

Sicherlich gibt es verschiedene Ansätze, grundlagenwissenschaftliche Daten und Fakten zu interpretieren. Die Autoren der folgenden Beiträge legen größten Wert darauf, dass hier vor allem eine Gesprächsbasis geschaffen werden soll, wohl wissend, dass umfangreiche Arbeiten im Sinne des „translational research“ hier noch erforderlich sind.

Die Umsetzung oben genannter Inhalte in der täglichen schmerztherapeutischen und funktionsmedizinischen Praxis ist jedoch so vielversprechend und so ermutigend, dass hier ein äußerst wichtiger Ansatz gesehen wird. Die Autorengruppe wünscht sich breites Interesse der praktisch tätigen Manualmediziner und ist für Anregungen aus dem Kreise der Kolleginnen und Kollegen für weitere Kooperationsansätze und geeignete Interpretationen dankbar.

\section{Eine weiter reichende Diskussion} anzustoßen und die Gesprächsbereitschaft in allen betroffenen Disziplinen zu fördern, ist das größte Anliegen.

Unter dem Stichwort „translationale Forschung" werden folgende thematischen Beiträge in den nächsten Heften der Manuellen Medizin zu finden sein (Arbeitstitel):

- Anatomie der afferenten Innervation

- unstrittige Tatsachen und Hypothesen im Grenzbereich (Teil 1 und 2)

- „Artikuläre Gesetze“ - wie mechanisch dürfen wir noch denken

- Die motorische Reflexantwort: ein multidimensional reguliertes komplexes System

- Rezeptive Felder - ihre Bedeutung für Diagnostik und Differenzialtherapie

- Die körpereigene Schmerzhemmung - ständig vorhanden, aber klinisch wenig beachtet

- Schmerz und Dysfunktion - supraspinale Aspekte

- Bedeutung des autonomen Nervensystems für den klinischen Alltag

Wir wünschen uns eine interessierte und engagierte Leserschaft, wohl wissend, dass manche Beiträge den Leser geradezu auf- fordern, auch wieder in Büchern früherer Phasen des Studiums (in den Neuauflagen!) nachzuschlagen. Das wird natürlich auch mit Aufwand verbunden sein. Mit der Erfahrung, dass diese neuen Erkenntnisse wieder zu sehr positiver Bestätigung in der täglichen Arbeit führen, wünschen die Autoren allen Lesern Motivation und Freude bei der Arbeit an und mit diesen Beiträgen.

\section{Korrespondenzadresse \\ Dr. H. Locher}

Zentrum für Schmerztherapie und manuelle Medizin

Lindauerstr. 16, 88069 Tettnang

derlocher@gmx.de

\section{Literatur}

1. Chila A, Fitzgerald M, American Osteopathic Association (2010) Foundations of osteopathic medicine, 3rd edn., Lippincott Williams \& Wilkins, Philadelphia

2. Goadsby PJ (2008) On the functional neuroanatomy of neck pain. Cephalgia 28 (Suppl 1):1-7

3. Handwerker HO (2007) Von Descartes bis zur fMRI. Schmerz 21:307-317

4. Handwerker HO (2010) Falsche Erwartungen an die Grundlagenforschung - oder erforschen wir das Falsche? MedReport 34:5

5. Locher H (2010) Die Schmerzanalyse bei Schmerzen am Bewegungsorgan und Ableitung einer rationalen Differentialtherapie. Praxisrelevante Assessments auf dem Boden grundlagenwissenschaftlicher Erkenntnisse. Orthop Praxis 46:57-74

6. Namer B, Handwerker HO (2009) Translational nociceptor research as guide to human pain perceptions and pathophysiology. Exp Brain Res 196:163-72 